\title{
Correction to: Platelet Proteomic Analysis Revealed Differential Pattern of Cytoskeletal- and Immune-Related Proteins at Early Stages of Alzheimer's Disease
}

\author{
Marta González-Sánchez ${ }^{1,2} \cdot$ Teresa Díaz $^{1}$ • Consuelo Pascual ${ }^{1}$ - Desiree Antequera ${ }^{1,2}$. \\ Alejandro Herrero-San Martín ${ }^{1,2}$. Sara Llamas-Velasco ${ }^{1,2} \cdot$ Alberto Villarejo-Galende $^{1,2} \cdot$ Fernando Bartolome $^{1,2}$. \\ Eva Carro ${ }^{1,2}$ (iD
}

Published online: 2 May 2020

(C) Springer Science+Business Media, LLC, part of Springer Nature 2020

\section{Correction to: Mol Neurobiol (2018) 55:8815-8825 https://doi.org/10.1007/s12035-018-1039-3}

The original version of this article unfortunately contained some mistakes.

In Figure 2A, the authors mistakenly used the same image for both the proteins Vinculin and Moesin. The authors apologize for this error and have provided the corrected Figure 2.

Also the following paragraph should be changed:

“...Blood samples were obtained through antecubital vein puncture. Platelets were isolated as described. Briefly, $12 \mathrm{ml}$ of blood from donor subjects was collected into $4.5-\mathrm{ml}$ tubes with $3.8 \%$ (wt/vol) sodium citrate, and samples were centrifuged at $200 \mathrm{~g}$ for $20 \mathrm{~min}$ at room temperature. Then, platelet-enriched plasma was centrifuged at $15000 \mathrm{~g}$ for $10 \mathrm{~min}$ at $4{ }^{\circ} \mathrm{C}$. Platelet pellets used for two-dimensional difference gel electrophoresis (2D-DIGE) and Western blot analysis were washed with cold phosphate-buffered saline (PBS) and centrifuged at $15000 \mathrm{~g}$ for $10 \mathrm{~min}$ at $4{ }^{\circ} \mathrm{C}$. Lately, platelets were resuspended in NP40 lysis buffer (50 mM Tris-base pH 7.4,

The online version of the original article can be found at https://doi.org/ 10.1007/s12035-018-1039-3

Fernando Bartolome

fbartolome.imas12@h12o.es

Eva Carro

carroeva@h12o.es

1 Group of Neurodegenerative Diseases, Hospital 12 de Octubre Research Institute (imas12), 28041 Madrid, Spain

2 Biomedical Research Networking Center in Neurodegenerative Diseases (CIBERNED), Madrid, Spain
$150 \mathrm{mM} \mathrm{NaCl}, 0.5 \%$ Nodidet P-40, 1 mM EDTA, protease and phosphatase inhibitor cocktails, Roche Applied Science) and stored at $-80^{\circ} \mathrm{C} . . . ”$

And the corrected paragraph is shown below:

“...Blood samples were obtained through antecubital vein puncture. Platelets were isolated as described. Briefly, $12 \mathrm{ml}$ of blood from donor subjects was collected into 4.5-ml tubes with $3.8 \%$ (wt/vol) sodium citrate, and samples were centrifuged at $200 \mathrm{~g}$ for $20 \mathrm{~min}$ at room temperature. Then, platelet-enriched plasma was centrifuged at $15000 \mathrm{~g}$ for $10 \mathrm{~min}$ at room temperature. Platelet pellets used for two-dimensional difference gel electrophoresis (2D-DIGE) and Western blot analysis were washed with phosphate-buffered saline (PBS) and centrifuged at $15000 \mathrm{~g}$ for $10 \mathrm{~min}$ at room temperature. Lately, platelets were resuspended in NP40 lysis buffer (50 mM Tris-base pH 7.4, $150 \mathrm{mM} \mathrm{NaCl}, 0.5 \%$ Nodidet P-40, 1 mM EDTA, protease and phosphatase inhibitor cocktails, Roche Applied Science) and stored at $-80^{\circ} \mathrm{C} . . . "$

Publisher's Note Springer Nature remains neutral with regard to jurisdictional claims in published maps and institutional affiliations. 